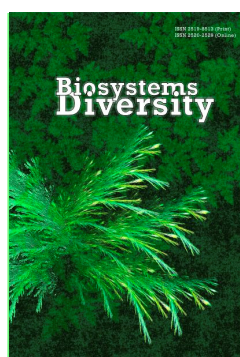

\title{
Chromosomal rearrangements caused by gamma-irradiation in winter wheat cells
}

\author{
M. M. Nazarenko, O. O. Izhboldin \\ Dnipropetrovsk State Agrarian and Economic University, Dnipro, Ukraine
}

Article info

Received 14.01.2017

Received in revised form 05.02.2017

Accepted 09.02.2017

Dnipropetrovsk State Agrarian and Economic University, Voroshilov str., 25 ,

Dnipro, 49600, Ukraine

Tel.: +38-095-848-53-86

E-mail:nik_nazarenko@ukr.net

\section{Nazarenko, M. M., \& Izhboldin, O. O. (2017). Chromosomal rearrangements caused by gamma-irradiation in winter wheat cells. Biosystems Diversity, 25(1), 25-28. doi: 10.15421/011704}

In this article we report the results of our investigation into several cytogenetic parameters of variability in mutation induction of modern winter wheat varieties and some connections between the means of cytogenetic indices and different doses of gamma-rays. Analysis of chromosomal aberrations following the action of any kind of mutagen by the anaphases method is one of the most widely investigated and most precise methods which can be used to determine the fact of mutagenic action on plants and identify the nature of the mutagen. We combined in our investigation the sensitivity of genotype to mutagen using cytological analysis of mutagen treated wheat populations with the corresponding different varieties by breeding methods to reveal its connections and differences, specific sensitivity to mutagens action on the cell level. Dry seeds of 8 varieties of winter wheat were subjected to 100, 150, 200, 250 Gy gamma irradiation, which are trivial for winter wheat mutation breeding. We investigated rates and spectra of chromosomal aberrations in the cells of winter wheat primary roots tips. The coefficients of correlations amid the rate of chromosomal aberrations and the dose of gamma-rays were on the level 0.8-0.9. The fragments/bridges ratio is a clear and sufficient index for determining the nature of the mutagen agent. We distinguished the following types of chromosomal rearrangements: chromatid and chromosome bridges, single and double fragments, micronuclei, and delayed chromosomes. The ratio of chromosomal aberrations changes with the change in mutagen; note that bridgetypes are characteristic of irradiation. Radiomutants are more resistant to gamma rays. This is apparent in the lower rate of chromosomal aberrations. Varieties obtained by chemical mutagenesis (varieties Sonechko, Kalinova) are more sensitive to gamma-irradiation than others. We propose these varieties as objects for a mutation breeding programme and radiation of mutants for planting in areas subject to the action of gamma-rays.

Keywords: chromosomal aberrations; gamma-rays; wheat; genotype

\section{Introduction}

There are three reasons for the study of plant mutant generation $\mathrm{M}_{1}$ after mutagenic effect. The first one is the idenfication of the suitability of agricultural land for growing major crops after pollution with mutagens. More than $70 \%$ of the soil in Ukraine used for agriculture is constantly exposed to chemical and physical mutagens. The second reason is the fact that the amount of material, obtained from the first generation, limits the opportunities for mutation breeding programmes (especially using lethal doses). The third reason is the use of obtained $\mathrm{M}_{1}$ population to extract valuable mutant strains in further generations.

Mutagenesis reduces plant growth and other crop yield structural components, increases the sterility of pollen and reduces the germination and survival abilities of plants by means of chemical agents and gamma rays; sometimes the greater part of a population is killed by critical doses (Solanki and Sharma, 2000). Depression increases with the increase of dose (Yilmaz and Erkan, 2006). Yet sometimes we observe a stimulating effect (in case of low doses) or absence of depression (at medium concentrations of some chemical mutagens). In the first generations of wheat as cultivar composed from three genomes we observed only some dominants. Positive desirable mutants can be selected and be incorporated in future breeding programmes (Ali Sakin et al., 2005).

Influence of different types of chemical mutagens or any type of ionizing radiation can be analyzed by the calculated number and types of chromosomal aberrations (Rakhmatullina, 2007). Chromosomal aberrations have long been recognized to be an important biomarker of living organisms' exposure to ionizing radiation and genotoxic chemicals. Change in both structure and the number of aberrations has been associated with problems in growth and development, e.g. congenital abnormalities in new-born living organisms. The rate of spontaneous chromosome aberrations is about $0.6 \%$ in plants. Both structural and numerical aberrations occur spontaneously due to internal and external factors (Nikolova et al., 2015).

The tendency of chromosomal rearrangements to cause visible mutations or modifications was first described in plants by de Vries (de Vries, 1918). Unlike other living organisms, plant systems offer an opportunity to detect the types and rates of chromosome changes in the first cell division following gamma-irradiation. It should be pointed out that these investigations were conducted long before the structure of DNA and chromosomes were known. A break in either single (resting stage) or divided (prophase) chromosomes is the consequence of direct action on the chromosome by the ionization produced by any elementary particle. Such a break may remain as such to give rise to terminal deletion, rejoin in the original position (restitution) or join with an indigenous break in the same or different chromosome to produce various types of rearrangements (Natarajan, 2002).

The quantitative relationship between the dose of radiation and the rate of aberration depends on both the type of rearrangements and the kind of radiation. With any type of radiation, elementary breaks have a linear relationship to the dose. Interchanges ascend with increasing of the dose. If a one-time dose of gamma-radiation is obtained, all the initial breaks are present simultaneously, reunion is disabled and the exchange produce has a squared proportion to 
the dose. If the dose is given chronically, restitution is disabled over the act of reunion and the exchange produce is decreased (Bolzarn and Bianchi, 2006).

Changes occur in chromosome number and structure in mitotic cells after irradiation. Chromosomal changes rank from breaks, through exchanges, laggards and anaphase bridges, dicentric and centric ring formations, terminal fragments with telomeric signal at only one end and interstitial fragments that appear as double minutes without any telomeric signals changes in irradiated mitotic cells (Rakhmatullina, 2007).

Joining with gene mutations, chromosomal aberrations in the form of different structure rebuilding (deletions, inversions, translocations, etc) were generates (Shu et al., 2011). Translocations are the most impotent for mutation breeding kind of changes on chromosome level, which possibly result from the rejoining of broken chromosome districts. Gamma-irradiation leads to generation of chromosome breaks, which can reunite in a random manner, the result of this process is translocation. Deletions are produced in the same way. Ionizing radiation generates cytogenetic aberrations and a large number of errors in chromosome segregation. Cytological aberrations observed in cell mitosis include the production of micronuclei and other types of chromosomal changes (Ukai, 2006; Shu et al., 2011).

Analysis of variability of chromosomal changes after mutagen treatment of any type of mutation factor by the anaphases method is one of the most widely investigated and precise methods which can be used for determining the fact of mutagen action on plants, and identifying the nature of mutagens (Lifang et al., 2001; Adlera et al., 2004; Ukai, 2006; Waugha, 2006). Moreover, this method is widely exploited for determining radionuclide pollution of land and water, its level and the danger of this pollution, for determining the optimal doses of radiation and concentrations of chemical supermutagens in breeding work with plant material (Ahloowalia et al., 2004; Nazarenko, 2015). Therefore, radiation mutation is a compound of high mutagenic, clastogenic and recombination activity in plants on the cell level, frequently stronger than that of the most powerful nitrosoalkylating agents (Grant and Owens, 2001). The relation between clastogenic adaptation shown in descent of chromatid type of changes, micronuclei and changes in the number of chromosomes in cells, and the clastogenic effect has been revealled (Bignold, 2005). The advantages of this approach are simplicity, objectivity of the results, reliability and the ability to make an integrated assessment of the impact of a wide variety of natural mutagens (Karthika and Subba, 2006; Albokari, 2014).

The main purpose of this investigation is evaluation of cytogenetic variability of mutation induction of chromosomal aberrations of modern wheat varieties and connections between the means of the cytogenetic characteristics and different doses of gamma-rays.

\section{Material and methods}

Winter wheat seeds (Triticum aestivum L.) (approximately 14\% moisture content) of (in brackets is given the method of obtaining the varieties or mutagens used) Favoritka, Lasunya, Hurtovina (irradiation by gamma rays), line 418, Kolos Mironovschiny (field hybridization), Sonechko and Kalinova (chemical mutagenesis), Voloshkova (thermomutagenesis - low plus temperature under vernalizaion was used as the mutagen factor) were subjected to 100 , $150,200,250$ Gy gamma rays. 1,000 wheat seeds were used for each treatment. These differences of doses are trivial for winter wheat mutation breeding (Ahloowalia et al., 2004; Nazarenko, 2015). Non-treated varieties were used as a control for each variety.

The seeds used in this study were of the $\mathrm{M}_{0}$ generation. After mutagen treatment, dry seeds were germinated in Petri dishes for 24-48 hours (depending on presoaking and mutagen action), at a temperature $+25^{\circ} \mathrm{C}$. Afterwards, the central primary roots were cut (provided their length was 10-15 mm) and fixed in a solution of alcohol and acetic acid (in proportion $3: 1$ ) for 24 hours. The fixation material was stored in $70 \%$ alcohol solution at a temperature $2{ }^{\circ} \mathrm{C}(30-35$ roots per variant). We conducted cytological analysis at temporary press-time preparations of primary root tips $(1.0-1.5 \mathrm{~mm}$ length) stained with acetocarmine (prepared by Remsderh). Tissue maceration was conducted with $45 \%$ solution of acetic acid (during 5 minutes at $60^{\circ} \mathrm{C}$ ). Anaphase of cell division was observed by light microscope JNAVAL. No less than 800 cells in proper phases of mitosis were observed in each variant (Lifang et al., 2001; Rank et al., 2002; Natarajan, 2005). Statistical analyis of dates obtained was conducted by the method of multi-factor analysis, the variability of the index difference was evaluated by ANOVA,the standard tools of the programme Statistica 8.0 were used. Standard error $( \pm \mathrm{SE})$ values of the treated variants are presented in Table 1.

Table 1

Rate of chromosomal aberrations in primary root cells of winter wheat

\begin{tabular}{|c|c|c|c|c|c|c|}
\hline \multirow{3}{*}{ Variant } & \multirow{2}{*}{$\begin{array}{l}\text { Mitosis, } \\
\text { number }\end{array}$} & \multicolumn{2}{|c|}{ Chromosomal aberrations } & \multirow{2}{*}{$\begin{array}{l}\text { Mitosis, } \\
\text { number }\end{array}$} & \multicolumn{2}{|c|}{ Chromosomal aberrations } \\
\hline & & number & percent & & number & percent \\
\hline & \multicolumn{3}{|c|}{ Favoritka } & \multicolumn{3}{|c|}{418} \\
\hline Control & 984 & 19 & $1.9 \pm 0.3$ & 962 & 11 & $1.1 \pm 0.1$ \\
\hline Gamma-rays, 100 Gy. & 1006 & 71 & $7.1 \pm 0.7^{*}$ & 992 & 161 & $16.2 \pm 1,1^{*}$ \\
\hline Gamma-rays, $150 \mathrm{~Gy}$ & 1004 & 139 & $13.9 \pm 1.1^{*}$ & 1056 & 245 & $23.2 \pm 1,2 *$ \\
\hline Gamma-rays, 200 Gy & 943 & 230 & $24.4 \pm 1.5^{*}$ & 747 & 228 & $30.5 \pm 1.6^{*}$ \\
\hline Gamma-rays, 250 Gy & 466 & 126 & $27.1 \pm 1.5^{*}$ & 586 & 247 & $42.2 \pm 1.9^{*}$ \\
\hline Variety & \multicolumn{3}{|c|}{ Lasunya } & \multicolumn{3}{|c|}{ Hurtovina } \\
\hline Control & 1056 & 15 & $1.4 \pm 0.2$ & 1034 & 12 & $1.2 \pm 0.1$ \\
\hline Gamma-rays, 100 Gy. & 979 & 88 & $9.0 \pm 0.8^{*}$ & 1012 & 100 & $9.9 \pm 0.9^{*}$ \\
\hline Gamma-rays, 150 Gy & 1012 & 158 & $15.6 \pm 1.1^{*}$ & 981 & 147 & $15.0 \pm 1.0^{*}$ \\
\hline Gamma-rays, 200 Gy & 810 & 198 & $24.5 \pm 1.5^{*}$ & 1011 & 228 & $22.6 \pm 1.5^{*}$ \\
\hline Gamma-rays, 250 Gy & 399 & 98 & $24.6 \pm 1.5$ & 742 & 193 & $26.0 \pm 1.6^{*}$ \\
\hline Variety & \multicolumn{3}{|c|}{ Sonechko } & \multicolumn{3}{|c|}{ Voloshkova } \\
\hline Control & 1026 & 8 & $0.8 \pm 0.1$ & 1003 & 31 & $3.1 \pm 0.3$ \\
\hline Gamma-rays, $100 \mathrm{~Gy}$. & 1010 & 194 & $19.2 \pm 1.1^{*}$ & 1000 & 213 & $21.3 \pm 1.2 *$ \\
\hline Gamma-rays, 150 Gy & 1003 & 288 & $28.7 \pm 1.3^{*}$ & 1007 & 332 & $33.0 \pm 1.4^{*}$ \\
\hline Gamma-rays, $200 \mathrm{~Gy}$ & 888 & 342 & $38.5 \pm 1.9^{*}$ & 560 & 266 & $47.5 \pm 2.0^{*}$ \\
\hline Gamma-rays, 250 Gy & 411 & 190 & $46.2 \pm 2.0^{*}$ & 478 & 198 & $41.4 \pm 1.8^{*}$ \\
\hline Variety & \multicolumn{3}{|c|}{ Kalinova } & \multicolumn{3}{|c|}{ Kolos Mironivschini } \\
\hline Control & 1047 & 9 & $0.9 \pm 0.1$ & 909 & 10 & $1.1 \pm 0.1$ \\
\hline Gamma-rays, 100 Gy. & 1000 & 192 & $19.2 \pm 1.1^{*}$ & 1019 & 179 & $17.6 \pm 1.0^{*}$ \\
\hline Gamma-rays, 150 Gy & 937 & 269 & $28.7 \pm 1.3^{*}$ & 890 & 215 & $24.2 \pm 1.2 *$ \\
\hline Gamma-rays, 200 Gy & 817 & 315 & $38.5 \pm 1.9^{*}$ & 738 & 243 & $32.9 \pm 1.7^{*}$ \\
\hline Gamma-rays, $250 \mathrm{~Gy}$ & 459 & 212 & $46.2 \pm 2.0^{*}$ & 510 & 196 & $38.4 \pm 1.8^{*}$ \\
\hline
\end{tabular}

Note: $*$ - difference is considered statistically significant at $\mathrm{P}<0.01$. 


\section{Results and discussion}

In Table 1 we represent the data of the results of our analysis with the following parameters: overall number of observed mitosis in primary root tips, number of cells with visible chromosomal aberrations, total rate of changes. As we can see from Table 1, frequencies of aberrations varied from $7.1 \%$ (Favoritka, gamma-rays $200 \mathrm{~Gy}$ ) to $47.5 \%$ (Voloshkova, gamma-rays $200 \mathrm{~Gy}$ ) of the total number of cell divisions. All the variants are statistically substantially dissimilar from each other and from the control (excluding $250 \mathrm{~Gy}$ ). A higher frequency of aberrations in any cases is characteristic for varieties obtained by chemical mutation breeding (Sonechko, Kalinova) and we can predict a higher rate of visible mutations (in view of previous investigations).

A higher frequency of aberrations was obtained by using the 200 Gy dose. Frequency was statistically lower when we used gammarays for the varieties Favoritka, Lasunya, Hurtovina obtained with gamma-irradiation. The same situation we observed for the varieties Kalinova and Sonechko, when NMU and NEU were used in our previous investigations, but with a more pronounced decrease in the case of gamma-rays. Thereby, the varieties Favoritka, Lasunya, Hurtovina are less sensitive to gamma-rays. Gamma-rays initiated a higher rate of chromosome aberrations than nitrosoalkylureas) (Nazarenko, 2016).

Table 2

Types of chromosomal aberrations in winter wheat

\begin{tabular}{|c|c|c|c|c|c|c|c|c|c|}
\hline \multirow[t]{2}{*}{ Variant } & \multicolumn{2}{|c|}{$\begin{array}{c}\text { Fragments } \\
\text { (single and double) }\end{array}$} & \multicolumn{2}{|c|}{$\begin{array}{l}\text { Bridges (chromosome } \\
\text { and chromatide) }\end{array}$} & \multirow{2}{*}{$\begin{array}{c}\text { Fragments / } \\
\text { bridges }\end{array}$} & \multicolumn{2}{|c|}{$\begin{array}{c}\text { Micronucleus, } \\
\text { lagging chromosomes }\end{array}$} & \multicolumn{2}{|c|}{ Complicated aberrations } \\
\hline & number & percent & number & percent & & number & percent & number & percent \\
\hline Favoritka, control & 6 & 54.6 & 5 & 45.5 & 1.2 & 0 & 0.00 & 2 & 18.2 \\
\hline Gamma-rays, 100 Gy & 17 & 23.9 & 51 & 71.8 & 0.3 & 3 & 4.2 & 5 & 7.0 \\
\hline Gamma-rays, 150 Gy & 28 & 20.1 & 101 & 72.7 & 0.3 & 10 & 7.2 & 21 & 15.1 \\
\hline Gamma-rays, 200 Gy & 67 & 29.1 & 152 & 66.1 & 0.4 & 11 & 4.8 & 38 & 16.5 \\
\hline Gamma-rays, 250 Gy & 52 & 41.3 & 71 & 56.4 & 0.7 & 3. & 2.4 & 34 & 27.0 \\
\hline Lasunya, control & 4 & 26.7 & 11 & 73.3 & 0.4 & 0 & 0.0 & 0 & 0.0 \\
\hline Gamma-rays, 100 Gy & 42 & 47.7 & 46 & 52.3 & 0.9 & 0 & 0.0 & 8 & 9.1 \\
\hline Gamma-rays, 150 Gy & 71 & 44.9 & 79 & 50.0 & 0.9 & 8 & 5.1 & 22 & 13.9 \\
\hline Gamma-rays, 200 Gy & 82 & 41.4 & 104 & 52.5 & 0.8 & 12 & 6.1 & 31 & 15.7 \\
\hline Gamma-rays, 250 Gy & 43 & 43.9 & 38 & 38.8 & 1.1 & 17 & 17.4 & 41 & 41.8 \\
\hline Hurtovina, control & 7 & 58.3 & 5 & 41.7 & 1.4 & 0 & 0.0 & 0 & 0.0 \\
\hline Gamma-rays, 100 Gy & 43 & 43.0 & 52 & 52.0 & 0.8 & 5 & 5.0 & 7 & 7.0 \\
\hline Gamma-rays, 150 Gy & 69 & 46.9 & 74 & 50.3 & 0.9 & 4 & 2.7 & 14 & 9.5 \\
\hline Gamma-rays, 200 Gy & 112 & 49.1 & 114 & 50.0 & 1.0 & 2 & 0.9 & 29 & 12.7 \\
\hline Gamma-rays, 250 Gy & 101 & 52.3 & 89 & 46.1 & 1.1 & 3 & 1.6 & 41 & 21.2 \\
\hline Line 418 , control & 6 & 54.6 & 5 & 45.5 & 1.2 & 0 & 0.0 & 2 & 18.2 \\
\hline Gamma-rays, 100 Gy & 58 & 36.0 & 101 & 62.7 & 0.6 & 2 & 1.2 & 6 & 3.7 \\
\hline Gamma-rays, 150 Gy & 81 & 33.1 & 143 & 58.4 & 0.6 & 21 & 8.6 & 31 & 12.7 \\
\hline Gamma-rays, 200 Gy & 64 & 28.1 & 132 & 57.9 & 0.5 & 32 & 14.0 & 38 & 16.7 \\
\hline Gamma-rays, 250 Gy & 101 & 40.9 & 132 & 53.4 & 0.8 & 14 & 5.7 & 52 & 21.1 \\
\hline Kolos Mironovschiny, control & 5 & 50.0 & 5 & 50.0 & 1.0 & 0 & 0.0 & 0 & 0.0 \\
\hline Gamma-rays, 100 Gy. & 73 & 40.8 & 101 & 56.4 & 0.7 & 5 & 2.8 & 12 & 6.7 \\
\hline Gamma-rays, 150 Gy & 81 & 37.7 & 122 & 56.7 & 0.7 & 12 & 5.6 & 18 & 8.4 \\
\hline Gamma-rays, 200 Gy & 92 & 37.9 & 138 & 56.8 & 0.7 & 13 & 5.4 & 32 & 13.2 \\
\hline Gamma-rays, 250 Gy & 78 & 39.8 & 116 & 59.2 & 0.7 & 2 & 1.0 & 28 & 14.3 \\
\hline Sonechko, control & 6 & 75.0 & 2 & 25.0 & 3.0 & 0 & 0.0 & 0 & 0.0 \\
\hline Gamma-rays, 100 Gy & 53 & 27.3 & 135 & 69.6 & 0.4 & 6 & 3.1 & 6 & 3.1 \\
\hline Gamma-rays, 150 Gy & 50 & 17.4 & 209 & 72.6 & 0.2 & 29 & 10.1 & 28 & 9.7 \\
\hline Gamma-rays, 200 Gy & 58 & 17.0 & 243 & 71.1 & 0.2 & 41 & 12.0 & 43 & 12.6 \\
\hline Gamma-rays, 250 Gy & 48 & 25.3 & 116 & 61.5 & 0.4 & 26 & 13.7 & 64 & 33.7 \\
\hline Kalinova, control & 2 & 22.2 & 7 & 77.8 & 0.3 & 0 & 0.0 & 0 & 0.0 \\
\hline Gamma-rays, 100 Gy & 80 & 41.7 & 112 & 58.3 & 0.7 & 0 & 0.0 & 29 & 15.1 \\
\hline Gamma-rays, 150 Gy & 100 & 37.2 & 164 & 61.0 & 0.6 & 5 & 1.9 & 42 & 15.6 \\
\hline Gamma-rays, 200 Gy & 112 & 35.6 & 193 & 61.3 & 0.6 & 10 & 3.2 & 64 & 20.3 \\
\hline Gamma-rays, 250 Gy & 81 & 38.2 & 127 & 59.9 & 0.6 & 4 & 1.9 & 37 & 17.5 \\
\hline Voloshkova, control & 16 & 51.6 & 13 & 41.9 & 1.2 & 2 & 6.5 & 5 & 16.1 \\
\hline Gamma-rays, 100 Gy & 101 & 47.4 & 108 & 50.7 & 0.9 & 4 & 1.9 & 18 & 8.5 \\
\hline Gamma-rays, 150 Gy & 142 & 42.8 & 174 & 52.4 & 0.8 & 16 & 4.8 & 35 & 10.5 \\
\hline Gamma-rays, 200 Gy & 108 & 40.6 & 143 & 53.8 & 0.8 & 15 & 5.6 & 67 & 25.2 \\
\hline Gamma-rays, $250 \mathrm{~Gy}$ & 47 & 23.7 & 136 & 68.7 & 0.4 & 15 & 7.6 & 43 & 21.7 \\
\hline
\end{tabular}

From the Table 1 we can see that the higher rates of chromosomal changes in any cases are characteristic for varieties obtained by mutation breeding with use of chemical mutagens (Sonechko, Kalinova). Gamma-rays were effective in aberration induction in case of chemical mutation varieties. Because of this, re-exposure of mutagens is acceptable as a method if we exploit other natural mutagens than first time (for example gamma-rays after nitrosoalkylureas in our pattern).

We developed the following types of aberrations of chromosomes after spectra investigation of: chromosomal bridges and double-bridges, fragments of chromosomes and double-fragments, micronucleus, lagging chromosomes. Cases with complicated aberrations (two or more kinds of changes in one mitosis) and ratio between fragments and bridges were counted singly (Table 2). The number of any type of chromosomal changes increased when the dose was raised (correlation coefficients were around $0.8-0.9$ ).
Previously we observed this evidence in our investigations when more bridges than fragments were induced with gamma-rays (fragments-bridges ratio lower than 1) (Nazarenko, 2016). However, more fragments and double-fragments were caused by chemical supermutagens (fragments-bridges ratio more than 1) (Nazarenko, 2015, 2016). We will be able to use this parameter to identify the action of gamma-rays action as similar to that of chemical mutagens but in opposite sense of parameter.

In general, when the dose of the mutagen was increased the frequency also increased. Also the percentage of complex (double and more changes in one cell) aberrations rose. On the other hand, complicated aberration occurs more frequently after NMU and NEU than after exposure to gamma-rays.

The results of three-factor analysis ("genotype", "dose" and "mutagen"; in the general scheme of analysis we include our data from previous investigation into the action of chemical mutagens 
(Nazarenko, 2016), show that the rate of chromosome aberrations was influenced most strongly by the "dose" factor, then by the "genotype" factor, then by the "mutagen" factor. However, the second and third factors involved more of the dispersion than the first. We found that repeated exposure to similar mutagens (for example, gamma-rays on the variety obtained by the action of this mutagenic factor) tends to produce a substantially lower rate of chromosomal aberrations.

Therefore, we propose this parameter (prevalence of fragments under bridges as fragments-bridges ratio) for identifying the type of mutagen. Complex (or combined) aberrations are more typical for chemical mutagens than for physical. Genotypes, obtained with gamma-rays are less sensitive to repeated action of the same mutagen. The previous rule for chemical supermutagens was confirmed for gamma-rays too. Gamma-rays will successful in mutation induction in case of application of for chemical mutations varieties.

\section{Conclusions}

Radiomutants are more resistant to gamma rays. This is apparent in the lower rate of chromosomal aberrations. We can predict a lower quantity of mutations if we exploit these varieties as mutation breeding objects.

Varieties obtained by chemical mutagenesis (varieties Sonechko, Kalinova) are more sensitive to gamma-irradiation than others (rates of chromosomal changes are higher). We propose these varieties as objects for a mutation breeding programme and radiation mutants for planting in areas subject to the action of gamma-rays action after pollution by radionuclide substances.

Comparison between bridges and fragments is a reliable index for identification of the nature of mutagens (chemical or gamma-rays). The highest numbers of any type of fragments were observed in the case of gamma-irradiation, the reverse applied with bridges. In general, the rate of any kind of chromosomal aberrations shows a linear increase with raised dose of the mutagen. Complex (or combined) aberrations are more typical for chemical mutagens than for physical.

\section{References}

Adlera, I., Carereb, A., \& Eichenlaub-Ritterc, U. (2004). Gender differences in the induction of chromosomal aberrations and gene mutations in rodent germ cells. Environmental Research, 17, 53-59.

Ahloowalia, B. S., Maluszynski, M., \& Nicoterlein, K. (2004). Global impact of mutation-derived varieties. Euphytica, 135, 187-204.

Albokari, M. (2014). Induction of mutants in durum wheat using gamma irradiation. Pakistan Journal of Botany, 46, 317-324.

Ali Sakin, M., Yildirim, A., \& Gikmen, S. (2005). Determining some yield and quality characteristics of mutants induced from a durum wheat
(Triticum durum Desf.) Cultivar. Turkean Journal Agriculture and Forestry, 29, 61-67.

Bignold, L. P. (2009). Mechanisms of clastogen-induced chromosomal aberrations: A critical review and description of a model based on failures of tethering of DNA strand ends to strand-breaking enzymes. Mutation Research, 681, 271-298.

Bolzarn, A. D., \& Bianchi, M. S. (2006). Telomeres, interstitial telomeric repeat sequences, and chromosomal aberrations. Mutation Research, 612, 189-214.

de Vries, H. (1918). Mass mutations and twin hybrids in Oenothera grandiflora Ait. Botanical Gazette, 65(5), 377-422.

Grant, W. F., \& Owens, E. T. (2001). Chromosome aberration assays in Pisum for the study of environmental mutagens. Mutation Research, 488, 93-118.

Karthika, I. R., \& Subba, B. (2006). Effect of gama rays and EMS on two varieties of soybean. Asian Journal of Biological Sciences, 5, 721-724.

Lifang, W., \& Zengliang, Y. (2001). Radiobiological effects of a low-energy ion beam on wheat. Radiation Environmental Biophysics, 40, 53-57.

Natarajan, A. T. (2002). Chromosome aberrations: Past, present and future. Mutation Research, 504, 3-16.

Natarajan, A. T. (2005). Chromosome aberrations: Plants to human and feulgen to FISH. Current Science, 89, 335-340.

Nazarenko, M. (2015). Negativnyie posledstviya mutagennogo vozdeystviya [Peculiarities of negative consequences of mutagen action]. Ecological Genetics, 4, 25-26 (in Russian).

Nazarenko, M. (2016). Parameters of winter wheat growing and development after mutagen action. Bulletin of Transilvania University of Brasov. Series II: Forestry, Wood Industry, Agricultural, Food Engineering, 58, 109-116.

Nazarenko, M. (2016). Specify of nitrosoalkylureas action on cell level in winter wheat. Visnyk of Dnipropetrovsk University. Biology, Ecology, 24(2), 258-263.

Nikolova, I., Georgieva, M., Kruppa, K., Molnor-Long, M., Liu, L., Manova, V., \& Stoilov, L. (2015). Cytogenetic effects in barley root apical meristem after exposure of dry seeds to lithium ion beams. Genetics and Plant Physiology, 5, 3-9.

Rakhmatullina, E. M., \& Sanamyan, M. F. (2007). Estimation of efficiency of seed irradiation by thermal neutrons for inducing chromosomal aberration in $\mathrm{M}_{2}$ of cotton Gossypium hirsutum L. Russian Journal of Genetics, 43(5), 518-524.

Rank, J., Lopez, L. C., \& Nielsen, M. H. (2002). Genotoxicity of maleic hydrazide, acridine and DEHP in Allium cepa root cells performed by two different laboratories. Hereditas, 136, 13-18.

Shu, Q. Y., Forster, B. P., \& Nakagava, H. (2011). Plant mutation breeding and biotechnology. CABI publishing, Vienna.

Solanki, I. S., \& Sharma, B. (2000). Significance and effectiveness of classifying the $\mathrm{M}_{1}$ material based on mutagenic damage for inducing macro- and micromutations in lentil. Indian Journal of Genetics and Plant Breeding, 60, 305-320.

Ukai, Y. (2006). Effectiveness and efficiency of mutagenic treatments: Gamma Field Symposia, 45, 1-4.

Waugha, R. (2006). Harvesting the potential of induced biological diversity. Trends in Plant Science, 11, 71-79.

Yilmaz, A., \& Erkan, B. (2006). The effects of Cobalt-60 applications on yield and yield components of cotton (Gossipium barbadense L.). Pakistan Journal of Biological Sciences, 22, 2761-2769. 